\title{
Diacronie
}

Studi di Storia Contemporanea

$\mathrm{N}^{\circ} 32,4 \mid 2017$

Proiezioni individuali e agire collettivo nella storia

\section{Le donne, il fordismo, Gramsci}

Una prospettiva di genere sulla società statunitense (1909-1930)

\section{Bruno Walter Renato Toscano}

\section{(2) OpenEdition \\ Journals}

Edizione digitale

URL: http://journals.openedition.org/diacronie/6670

DOI: 10.4000/diacronie.6670

ISSN: 2038-0925

Editore

Association culturelle Diacronie

Notizia bibliografica digitale

Bruno Walter Renato Toscano, « Le donne, il fordismo, Gramsci », Diacronie [Online], N 32, 4| 2017, documento 6, Messo online il 29 décembre 2017, consultato il 30 avril 2019. URL : http://

journals.openedition.org/diacronie/6670; DOI : 10.4000/diacronie.6670 


\title{
Diacronie
}

Studi di Storia Contemporanea

$32,4 / 2017$

Proiezioni individuali e agire collettivo nella storia. Ruoli sociali, aspetti politici e nodi storiografici tra pubblico e privato

\section{Le donne, il fordismo, Gramsci: una prospettiva di genere sulla società statunitense (1909-1930)}

\author{
Bruno Walter Renato TOSCANO
}

Per citare questo articolo:

TOSCANO, Bruno Walter Renato, «Le donne, il fordismo, Gramsci: una prospettiva di genere sulla società statunitense (1909-1930)», Diacronie. Studi di Storia Contemporanea : Proiezioni individuali e agire collettivo nella storia. Ruoli sociali, aspetti politici e nodi storiografici tra pubblico e privato, 32, 4/2017, 29/12/2017,

URL: < http://www.studistorici.com/2017/12/29/toscano_numero_32/ >

Diacronie Studi di Storia Contemporanea $\rightarrow$ http://www.diacronie.it

Rivista storica online. Uscita trimestrale.

redazione.diacronie@hotmail.it

Comitato di direzione: Naor Ben-Yehoyada - João Fábio Bertonha - Christopher Denis-Delacour - Maximiliano Fuentes Codera Anders Granås Kjøstvedt - John Paul Newman - Deborah Paci - Niccolò Pianciola - Spyridon Ploumidis - Wilko Graf Von Hardenberg

Comitato di redazione: Jacopo Bassi - Luca Bufarale - Gianluca Canè - Fausto Pietrancosta - Alessandro Salvador - Matteo Tomasoni - Luca Zuccolo

Diritti: gli articoli di Diacronie. Studi di Storia Contemporanea sono pubblicati sotto licenza Creative Commons 3.0. Possono essere riprodotti e modificati a patto di indicare eventuali modifiche dei contenuti, di riconoscere la paternità dell'opera e di condividerla allo stesso modo. La citazione di estratti è comunque sempre autorizzata, nei limiti previsti dalla legge. 


\title{
6/ Le donne, il fordismo, Gramsci: una prospettiva di genere sulla società statunitense (1909-1930)
}

\author{
Bruno Walter Renato TOSCANO
}

\begin{abstract}
Uno dei temi meno esaminati all'interno dell'analisi del fordismo è certamente quello riguardante le donne. Sebbene siano state scritte alcune pubblicazioni al riguardo, molte delle quali hanno trattato il tema indirettamente, non si è delineata una ricerca completa circa il tema in questione. Questo articolo tenterà di analizzare la relazione tra il fordismo e i rapporti di genere - sia interni che esterni alla fabbrica automobilistica - nel periodo che comprende gli anni Dieci del Novecento e la fase subito precedente allo scoppio della Seconda Guerra. Si farà qui particolare riferimento all'analisi di Gramsci presente nei Quaderni del Carcere sul concetto di fordismo, ma anche sulla sua interpretazione di americanismo, taylorismo e di «quistione sessuale», evidenziando così il ruolo della donna attraverso tre figure talvolta distinte, molto spesso confuse tra loro: la donna come operaia, sottoposta a determinate politiche industriali; la donna come parte del processo di razionalizzazione messo in atto da Henry Ford; la donna come consumatrice all'interno del mercato automobilistico delle industrie Ford.
\end{abstract}

\section{Introduzione}

La riflessione sul ruolo della donna all'interno della produzione fordista è avvenuta soltanto negli anni Settanta, quando tale sistema economico ha iniziato ad essere analizzato a partire dal suo superamento, cioè con l'iniziale teorizzazione del concetto di postfordismo. Con tale termine, sviluppatosi all'interno delle sinistre operaiste all'inizio del decennio ${ }^{1}$, intendiamo non più un sistema produttivo incentrato sul lavoro ripetitivo di massa, ma una fase industriale contraddistinta dall'uso di nuove tecnologie e nuovi criteri organizzativi. Il fordismo è stato così descritto a partire da elementi parzialmente estranei ad esso, come il concetto di flessibilità, che diventa maggiormente rilevante successivamente al periodo fordista, o la relazione tra i sindacati

\footnotetext{
${ }^{1}$ Cfr. SETTIS, Bruno, Fordismi. Storia politica della produzione di massa, Bologna, Il Mulino, 2016, p. 20.
} 
e la fabbrica ${ }^{2}$. Come afferma Bruno Settis «[il fordismo] forse non è stato capito ancora oggi: rimane un problema storico aperto ${ }^{3}$. Come evidenziato dalla geografa Linda McDowell, le analisi sul passaggio dal Fordismo al post-Fordismo condotte da varie scuole di pensiero - come la scuola francese degli economisti regolazionisti e l'americana flexible specialization - non misero in luce il ruolo della donna all'interno della produzione di massa, se non per sottolinearne l'irrilevanza nel processo produttivo ${ }^{4}$. Specialmente Aglietta, rappresentante dei regolazionisti, considerò il ruolo della donna nel meccanismo fordista in riferimento alla sola sfera privata ${ }^{5}$.

Sebbene sia stato ampiamente utilizzato lo schema gramsciano di americanismo e fordismo circa il processo industriale di massa ${ }^{6}$, non è però stato approfondito in un'ottica di genere, soprattutto per quanto riguarda la relazione tra la «quistione sessuale» ${ }^{7}$ descritta da Gramsci e il lavoro delle operaie. Un tentativo è stato fatto dalla storica Laura E. Ruberto, con il libro Gramsci, Migration, and the Representation of Women's Work in Italy and the U.S. ${ }^{8}$, dove vengono utilizzate le teorie di Gramsci per descrivere la rappresentazione culturale e sociale delle donne italiane emigrate in America e delle lavoratrici italiane.

Nonostante Ruberto non si soffermi ad analizzare il lavoro di fabbrica, questo libro pioneristico cerca di colmare il vuoto esistente nella letteratura circa il rapporto tra il lavoro delle donne e lo schema gramsciano. In questo senso, per rendere produttivo questo legame, diventa necessario comprendere cosa intenda Gramsci per fordismo, e come questo si leghi ad un'analisi di genere, oltre a comprendere come le teorie di Gramsci si pongano come elemento di riconfigurazione all'interno del concetto di classe di matrice marxista, considerando così elementi che dal marxismo classico vennero descritti solo in virtù del loro rapporto con la struttura economica 9 . Pertanto, mentre nel primo capitolo di questo articolo ci concentreremo sulla analisi di Gramsci presente all'interno dei Quaderni del carcere (soprattutto il Quaderno 22, intitolato Americanismo e Fordismo), nei restanti paragrafi cercheremo di applicare tali concetti ad un'analisi approfondita dei rapporti sociali, lavorativi e di forza subiti o messi in atto dalle donne all'interno e all'esterno della fabbrica Ford.

Il termine "genere" qui utilizzato si basa sulla intramontabile definizione nata dalle ricerche della storica statunitense Joan W. Scott, composta da due proposizioni distinte ma che

\footnotetext{
${ }^{2}$ Cfr. McDOWELL, Linda, "Life without father and Ford: the new gender order of post-Fordism», in Transactions of the Institute of British Geographers, 16, 4/1991, pp. 400-419, p. 402; SETTIS, Bruno, op. cit., p. 22.

${ }^{3}$ SETTIS, Bruno, op. cit., p. 23.

${ }^{4}$ Cfr. McDOWELL, Linda, op. cit, pp. 403-405.

${ }^{5} \mathrm{Cfr}$. ibidem.

${ }^{6}$ Come evidenziato da Settis, l'influenza di questo schema si nota moltissimo in Operai e capitale di Tronti (TRONTI, Mario, Operai e capitale, Roma, Deriveapprodi, 2006). SETTIS, Bruno, op. cit., p. 20.

${ }^{7} \mathrm{La}$ «quistione sessuale» ricorre a più riprese nei Quaderni del carcere di Gramsci.

${ }^{8}$ RUBERTO, Laura E., Gramsci, Migration, and the Representation of Women's Work in Italy and the U.S., London, Lexington Book, 2007.

${ }^{9}$ È il caso della questione razziale e delle differenze culturali tra le classi. Cfr. ibidem, p. 8.
} 
interagiscono potentemente tra loro: «Il genere è un elemento costitutivo delle relazioni sociali fondate su una cosciente differenza tra i sessi, e il genere è un fattore primario del manifestarsi dei rapporti di potere» ${ }^{10}$.

Oltre alle fonti secondarie, l'articolo si avvale di fonti primarie come pamphlet pubblicitari prodotti dalla fabbrica Ford, articoli di giornali coevi al periodo preso in esame e foto d'epoca, utilizzate per descrivere le diverse rappresentazioni della donna all'interno del mercato automobilistico.

\section{Il Fordismo e la «quistione sessuale» come superstruttura}

Il Quaderno Ventidue, elaborato principalmente nella «primissima fase di elaborazione dei Quaderni» ${ }^{11}$ da Gramsci (quindi all'incirca tra il 1929 e il 1930), non si presenta come un semplice esame delle pratiche impiegate da Ford per la produzione di massa, ma si manifesta come struttura analitica che connette il concetto di egemonia a una rilettura del capitalismo attraverso l'americanismo, «fenomeno complesso» che si palesa essere sia sviluppo della razionalizzazione del capitalismo moderno, sia «il maturare di processi parassitari immanenti a quello stesso sviluppo» ${ }^{12}$.

In questo senso, Gramsci descrive un'economia industriale programmatica ${ }^{13}$, basata sulla supremazia dell'industria sulla campagna ${ }^{14}$ che diventa totale all'interno della società nordamericana, in quanto cerca di razionalizzare non solo l'attività produttiva, ma la vita sociale degli individui, sussumendo quest'ultima all'interno del processo lavorativo.

Ciò si dà, afferma il filosofo, grazie a quell'egemonia che «nasce dalla fabbrica e [che] non ha bisogno di intermediari», ovvero una struttura che domina le soprastrutture semplificandole ${ }^{15}$. La razionalizzazione dell'egemonia americanista esposta da Gramsci produce delle conseguenze sociali non indifferenti: si ritiene necessario elaborare antropologicamente un «nuovo tipo umano», un soggetto «conforme al nuovo tipo di lavoro e di processo produttivo» ${ }^{16}$. Ciò che si esterna in un esperimento, un tendere $a$, in vista della creazione di questo nuovo modello di lavoratore - che come vedremo si vuole sostanzialmente de-umanizzato, cioè privato della propria personalità psico-fisica - viene visto dal pensatore sardo come tentativo idilliaco della potenza

\footnotetext{
${ }^{10}$ SCOTT, Joan W., Il "genere": un'utile categoria di analisi storica, in ID., Genere, politica, storia, Roma, Viella, 2013, pp. 31-63.

${ }^{11}$ BARATTA, Giorgio, Americanismo e fordismo, in FROSINI, Fabio, LIGUORI, Giorgio (a cura di), Le parole di Gramsci. Per un lessico dei Quaderni del Carcere, Roma, Carocci, 2004, p. 17.

${ }^{12}$ Ibidem, p. 33.

${ }^{13}$ Cfr. GRAMSCI, Antonio, Quaderni del Carcere, Torino, Einaudi, 2014, p. 2139.

${ }^{14}$ Cfr. BARATTA, Giorgio, op. cit., p. 22.

${ }^{15}$ Cfr. GRAMSCI, Antonio, op. cit., p. 2146.

${ }^{16}$ Ibidem.
} 
americana, quest'ultima basata sulla sua «"verginità" storico-culturale» rispetto alle altre realtà europee ad essa contemporanea, che ne determina l'assenza sia delle «incrostazioni parassitarie» che delle «sedimentazioni passive» ancora presenti nel vecchio mondo ${ }^{17}$.

La distinzione operante tra nuovo e vecchio mondo, tra l'Occidente dell'egemonia americanista e il resto del mondo non ancora razionalizzato ${ }^{18}$, si intravede anche in quella che Gramsci chiama «quistione sessuale». Quest'ultima è una delle tante sfaccettature del fordismo, il quale tenta a più gradi di soggiogare «l'elemento "animalità" dell'uomo», obiettivo verso cui l'industrialismo, secondo il filosofo, ha sempre puntato, attraverso una pressione coercitiva su tutto il corpo sociale, limitando gli istinti naturali dell'uomo al fine di rendere sempre più perfetto il lavoro produttivo $^{19}$. A tal fine la "quistione sessuale» viene definita come parte di una superstruttura, concetto che, come nella sovrastruttura di Marx, «ingloba anche le istituzioni» attraverso cui la classe superiore domina le classi subalterne, diffondendo le proprie ideologie attraverso non solo elementi come il folklore o l'arte, ma anche le strutture adibite a diffondere il sapere, come biblioteche o scuole ${ }^{20}$. Il legame tra la repressione degli istinti animaleschi dell'operaio e la «quistione sessuale» si legano, dove il primo elemento ingloba il secondo nel controllo capillare della classe dei lavoratori, «organizzando [i loro] corpi nel tempo e nello spazio» ${ }^{21}$.

Sebbene Gramsci ammetta che la «quistione sessuale», grazie ai progressi dell'igiene, sia «un elemento a sé stante della quistione economica» ${ }^{22}$, essa sta comunque alla base di una analisi in cui si ritiene necessario spiegare come il sorgere del fordismo abbia richiesto la razionalizzazione della sessualità della classe operaia, la quale ha interagito con i ruoli di genere. In questo senso si inserisce il problema sociale a cui è andata incontro la sfera delle donne: se da una parte le proletarie vivono i contraccolpi della razionalizzazione industriale di Ford, dall'altra le donne delle classi elevate vengono rappresentate come oggetto di una sessualità urbana diventata uno «sport» ${ }^{23}$.

Ma è necessario contestualizzare in quale momento storico si inserisce il presente discorso: il filosofo si riferisce ad una crisi della moralità delle classi elevate, nella fattispecie della «donna “americanizzata" $»^{24}$, estetizzata a tal punto dal cinema, dai concorsi di bellezza e dal teatro, da

\footnotetext{
${ }^{17}$ Ibidem; cfr. anche BARATTA, Giorgio, op. cit. p. 18.

${ }^{18}$ Cfr. GRAMSCI, Antonio, op. cit., p. 2145.

${ }^{19} \mathrm{Cfr}$. ibidem, pp. 2160-2161.

${ }^{20}$ Cfr. GRISONI, Dominique, MAGGIORI, Robert, Guida a Gramsci, Milano, Bur, 1975, p. 253. Per un approfondimento circa il problema della superstruttura e la sua eredità marxista, cfr. COSPITO, Giuseppe, Struttura-superstruttura, in FROSINI, Fabio, LIGUORI, Giorgio (a cura di), op. cit., pp. 227-246.

${ }^{21}$ FRASER, Nancy, «From discipline to flexibilization? Rereading Foucault in the shadow of globalization», in Constellations, 2/2003, pp. 160-171, p. 162.

${ }^{22}$ GRAMSCI, Antonio, op. cit., p. 2149.

${ }^{23}$ Ibidem, p. 2148.

${ }^{24}$ BARATTA, Giorgio, op. cit., p. 23.
} 
diventare un «mammifero di lusso» ${ }^{25}$, in un contesto in cui la pubblicizzazione dilagante negli Stati Uniti usa le donne come modello pubblicitario anche per le prime automobili, rappresentando maggiormente donne di classe elevata ${ }^{26}$. Sebbene quest'ultime vivono senza restrizioni sociali, «attraversando continuamente l'oceano» e contraendo dei «matrimoni stagionali» che - per opinione di Gramsci - appaiono come una «prostituzione larvata dalle formalità giuridiche», le donne delle classi inferiori si trovano divise tra le politiche proibizioniste statunitensi ${ }^{27}$ e la costrizione da parte delle classi superiori a una «moralità-costume», depravandole ${ }^{28}$.

Per quanto questo legame tra le politiche fordiste in ambito economico-produttivo e le sue ricadute sociali, possa sembrare secondario, in realtà non lo è affatto. Anzi, è la produttività stessa che lo richiede: i controlli della Ford sulla vita degli operai, attraverso il Sociological Department $^{29}$, sono la dimostrazione di uno «sforzo collettivo» per far operare il «tipo nuovo di lavoratore», che non occupi la propria mente se non con il lavoro meccanico e automatizzato ${ }^{30}$. In termini hegeliani, il lavoro dell'operaio viene alienato, giacché: «quanto più si meccanizza il lavoro, tanto più ne diminuisce, il valore e tanto più l'individuo diventa un mero strumento [...] Le facoltà dell'individuo si restringono all'infinito, e la coscienza del lavoratore nella fabbrica degrada al più basso livello di monotonia» ${ }^{31}$.

Per bilanciare l'alienazione a cui è sottoposto l'operaio, gli industriali applicano la politica degli alti salari, combinando così persuasione e coercizione; in più si rende necessario che il salario percepito dal lavoratore venga speso «razionalmente», cioè evitando una emorragia di denaro in attività rese illecite dal proibizionismo, ovvero la prostituzione e l'alcool ${ }^{32}$.

Attraverso il controllo degli stipendi, attraverso una politica privata che Gramsci teme possa diventare «ideologia statale», si favorisce una politica proibizionista, evitando che il lavoratore alienato incontri la depravazione ${ }^{33}$. Si capisce quindi come la «quistione sessuale», come elemento superstrutturale, diventi integrante nella razionalizzazione del lavoratore: «L'operaio che va al lavoro dopo una notte di "stravizio" non è un buon lavoratore, l'esaltazione passionale non può andar d'accordo coi movimenti cronometrati dei gesti produttivi legati ai più perfetti automatismi $»^{34}$.

\footnotetext{
${ }^{25}$ GRAMSCI, Antonio, op. cit., p. 492.

${ }^{26}$ Cfr. GUDIS, Catherine, Buyways. Billboards, Automobiles, and the American Landscape, New York, Routledge, 2004.

${ }^{27}$ GRAMSCI, Antonio, op. cit., p. 492.

${ }^{28}$ Cfr. ibidem, pp. 490, 2162; cfr. anche BARATTA, Giorgio, op. cit., p. 23.

${ }^{29}$ Cfr. SETTIS, Bruno, op. cit., p. 76.

${ }^{30}$ GRAMSCI, Antonio, op. cit., p. 2166.

${ }^{31}$ DUNAYEVSKAYA, Raya, Marxismo e libertà, Firenze, La Nuova Italia, 1962, p. 11.

${ }^{32}$ Cfr. GRAMSCI, Antonio, op. cit., p. 2166.

${ }^{33}$ Cfr. ibidem, pp. 2166-2167.

${ }^{34}$ Ibidem.
} 
Ma che ruolo gioca la donna in questo contesto? Sebbene nelle trattazioni di Gramsci essa occupi un ruolo di primo piano dal punto di vista sociale, bisogna adesso chiedersi se all'"uomo nuovo" nato dalla razionalizzazione industriale, corrisponda la formazione di una "nuova donna", cioè se le donne trovino o meno posto all'interno delle industrie Ford.

\section{Le operaie della Ford: esclusione o integrazione?}

La presenza delle operaie all'interno delle fabbriche Ford tra gli anni Dieci e gli anni Quaranta del Novecento si aggira mediamente intorno all' $1,2 \%^{35}$, non rappresentando quindi una parte consistente della forza lavoro della industria automobilistica. Ciò palesa fin dall'inizio una loro esclusione quasi totale da parte del processo di produzione industriale delle fabbriche automobilistiche. Ma ciò basta ad affermare che il ruolo della donna sia stato irrilevante per le politiche industriali applicate da Ford? A questa domanda bisogna rispondere negativamente: sebbene le donne vengano incluse in un periodo successivo all'interno delle politiche industriali della nota casa automobilistica, escludendole inizialmente dalla politica degli alti salari del $1914^{36}$, esse comunque costituiscono una parte essenziale delle strategie messe in atto dall'industriale. In più, come afferma Ava Baron, l'importanza dell'analisi di genere non si basa solo sulla presenza effettiva delle donne, ma diventa importante anche nel considerare la loro assenza ${ }^{37}$.

Intanto bisogna capire da chi fosse costituito quell'1,2\%: sono donne non coniugate, vedove, o mogli di invalidi incapaci di poter provvedere al mantenimento della famiglia ${ }^{38}$. Questa esclusione delle donne sposate derivava da quella politica sociale fordista che già Gramsci aveva compreso: si doveva creare un uomo nuovo, razionalizzato in tutto, compresa la famiglia; pertanto si relegavano alla donna i compiti domestici e la cura dei bambini, così da lasciare all'uomo il ruolo di breadwinner, mascolinizzando il lavoro di fabbrica. Come sottolinea Alessandra Pescarolo:

Di fatto la norma del breadwinner si generalizza e ha un successo crescente nel razionare il lavoro, creando una fase di piena occupazione dei maschi adulti. L'esclusione delle mogli dal lavoro operaio, buono ma scarso, implica il dimezzamento della forza lavoro adulta e consente la crescita dei salari dei capifamiglia ${ }^{39}$.

\footnotetext{
${ }^{35}$ Dalla media aritmetica dei dati presenti nella tabella 2, Female Employment at Ford Motor Company (19121941), in LEWCHUCK, Wayne A., «Men and Monotony: Fraternalism as a Managerial Strategy at the Ford Motor Company", in The Journal of Economic History, 53, 4/1993, pp. 824-856, p. 830.

${ }^{36}$ Cfr. MAY, Martha, «The Historical Problem of the Family Wage: The Ford Motor Company and the Five Dollar Day», in Feminist Studies: Women and Work, 8, 2/1982, pp. 399-424, p. 413; LEWCHUCK, Wayne, A., op. cit., p. 840.

${ }^{37}$ Cfr. BARON, Ava, Workengendered: Toward a new history of American labor, New York, Cornell University Press, 1991, p. 20.

${ }^{38}$ MAY, Martha, op. cit, p. 413; LEWCHUCK, Wayne, A., op. cit., p. 847.

${ }^{39}$ PESCAROLO, Alessandra, Genere e famiglia in età contemporanea, in CALVI, Giulia (a cura di), Innesti, Roma,
} 
In buona sostanza, le uniche operaie possibili erano quelle che non avevano - o non avevano ancora - un uomo a cui affidare il compito di guadagnare il necessario per la famiglia. Come ricorda Settis, il Profit-sharing Plan - o five dollar day - definiva le donne «fattori economici secondari e inaffidabili, in quanto rischiavano di andarsene da un giorno all'altro a causa di matrimoni o gravidanze improvvise $»^{40}$, rendendo quindi restia l'industria ad assumere personale femminile.

Tuttavia, il numero delle operaie coniugate crebbe nel tempo nelle fabbriche Ford, con un picco durante la Seconda guerra mondiale, a causa dell'assenza temporanea dei mariti costretti ad arruolarsi dall'entrata degli USA nel conflitto, diminuendo poi progressivamente a partire dal $1946^{41}$, così come accadrà in gran parte del mercato del lavoro ${ }^{42}$. Ma per gli anni da noi compresi, le limitazioni poste alle donne non furono poche. Quando Ford decise di applicare la politica degli alti salari, raddoppiando lo stipendio base di un operaio attraverso il famoso five dollar day del 1914, destinato ad ulteriori incrementi in risposta alla crisi economica della fine degli anni Venti $^{43}$, in un primo periodo le donne furono escluse, provocando le ire di alcune femministe dello stabilimento di Highland Park ${ }^{44}$.

In realtà, l'iniziale aumento dei salari non copriva il totale dei lavoratori delle fabbriche automobilistiche e questo perché il lavoratore doveva possedere determinati requisiti, tra cui lavorare per la Ford da almeno sei mesi, avere più di ventidue anni, e in più essere sposati o badare ad una madre vedova così come era necessaria la conoscenza della lingua inglese ${ }^{45}$. Nonostante tutto, come sostiene Martha May, ciò non impediva ai lavoratori dell'industria di sentirsi parte di una grande compagnia che li spingeva a formare delle famiglie stabili in cui ognuno doveva occupare un ruolo fisso ${ }^{46}$. Raddoppiare il salario di un operaio voleva dire, da una parte, razionalizzare la classe operaia, mentre dall'altra poneva delle differenze di genere soprattutto all'interno della sfera privata dei lavoratori. Gramsci riconosce che all'industriale importava soltanto rendere le proprie maestranze efficienti e stabili, quindi l'uso del five dollar day

Viella, 2004, pp. 223-255, p. 239.

${ }^{40}$ SETTIS, Bruno, op. cit., p. 76.

${ }^{41}$ Cfr. KOSSOUDJI, Sherrie A., DRESSER, Laura J., «Working Class Rosies: Women Industrial Workers during World War II», in The Journal of Economic History, 52, 2/1992, pp. 431-446, pp. 434-435.

${ }^{42}$ Cfr. WEINER, Lynn Y., From Working Girl to Working Mother. The Female Labor Force in the United States, 18201980, London, The University of North Carolina Press, 1985, p. 89.

${ }^{43}$ Cfr. SETTIS, Bruno, op. cit., p. 85.

${ }^{44}$ Cfr. NEVINS, Allan, HILL, Frank E., Ford. The Times, the man, the Company, New York, Charles Scribner's Sons, 1954, p. 547.

${ }^{45}$ CLARKE, Deborah, Driving Women. Fiction and Automobile Culture in Twentieth-Century America, Baltimore, The Johns Hopkins University Press, 2007, p. 47; RAUSHENSBUSH, Carl, Fordism. Ford and the workers, Ford and the community, New York, League for Industrial Democracy, 1937, p. 11. May afferma che solo all' $80 \%$ dei lavoratori maschi sarebbe stato raddoppiato lo stipendio. MAY, Martha, op. cit., p. 413.

${ }^{46}$ Cfr. MAY, Martha, op. cit., p. 413. 
gli garantiva la possibilità di bloccare il turn-over, perché «anche il complesso umano [...] di un'azienda è una macchina che non deve essere troppo spesso smontata e rinnovata nei suoi pezzi singoli senza perdite ingenti $»^{47}$.

D'altra parte, la decisione di Ford di rendere il five dollar day parte del cosiddetto family wage, poneva una divisione di genere notevole; come spiega Lawrence Glickman, tale termine si riferisce a quelle teorie nate durante la progressive era, le quali affermavano l'esigenza di dover dare alla classe operaia uno stipendio con il quale il capofamiglia avrebbe dovuto sostenere il peso dell'intera famiglia, risollevando così la situazione di molte delle famiglie povere americane ${ }^{48}$.

Non fu una decisione priva di conseguenze: si determinarono famiglie strutturate a partire dalla figura del breadwinner, a cui spettava l'onere di guadagnare per tutta la famiglia, escludendo la moglie dal mercato del lavoro. In questo senso venne rafforzato il sistema patriarcale, limitando la donna in maniera duplice: l'operaia Ford non venne coinvolta nella politica degli alti salari e non vide mai le stesse agevolazioni riservate agli operai maschi ${ }^{49}$, mentre le mogli degli operai venivano considerate adatte solo alla cura della casa e dei figli. Entrambe venivano implicitamente considerate incapaci di potere mantenere economicamente la famiglia, ritenendone in grado solo il marito. In questo senso, nota May, gli studi sulla povertà condotti nel primo ventennio del Novecento rivelarono che molte delle famiglie povere erano rette da donne: fu così che uno strumento nato principalmente per combattere la povertà dilagante tra gli strati sociali più bassi, divenne un modo per "perpetuare la dipendenza e la povertà delle donne» ${ }^{50}$.

La donna diventa importante in quanto parte di quel discrimine tra ciò che costituisce un universo prevalentemente maschile, il lavoro di fabbrica (indipendente, ripetitivo e remunerativo), e un universo esclusivamente femminile, la domesticity (dipendente e non pagato). Questa divisione si riflette così nelle pressioni razionaliste di Ford, il quale, attraverso il Sociological Department, non solo poté controllare in maniera capillare se i vizi entrassero o meno nelle case degli operai ma, grazie a tale sistema di controllo, così ebbe modo di accertarsi che il solo stipendio "guadagnato da un operaio maschio» fosse sufficiente per i bisogni di una «famiglia economicamente dipendente» ${ }^{51}$. Pertanto, si può dire che lo scopo di Henry Ford non fu quello di escludere le donne, ma realizzare un modello familiare determinato sia nei rapporti di genere che nei rapporti lavorativi, da cui far nascere utopicamente una classe operaia felice, stabile, dedita al consumismo, grazie al supporto di un avvocato pagato dall'azienda «per aiutare i

\footnotetext{
${ }^{47}$ GRAMSCI, Antonio, op. cit., p. 2166.

${ }^{48}$ Cfr. GLICKMAN, Lawrence B., A Living Wage. American Workers and the Making of Consumer Society, New York, Cornell University Press, 1997, p. 158.

${ }^{49}$ Cfr. SETTIS, Bruno, op. cit., p. 76. Ciò vale per molte delle industrie più importanti dell'epoca, come spiegato in KESSLER-HARRIS, Alice, Gendering Labor History, Urbana-Chicago, University of Illinois Press, 2007, p. 162.

${ }^{50}$ MAY, Martha, op. cit. p. 405.

${ }^{51}$ Ibidem, p. 415.
} 
lavoratori a contrarre prestiti sicuri e mutui per nuove case $»^{52}$, ma soprattutto libera da qualsiasi sindacato. Quest'ultimo avrebbe influito negativamente sulla stabilità del rapporto filiale tra l'imprenditore statunitense e i lavoratori.

D'altra parte, la politica degli alti salari garantì a Ford la possibilità di rendere se stesso un altruista agli occhi della classe dei lavoratori, soprattutto agli occhi della società civile: è esplicito in uno dei quotidiani di Cambridge attivi fino alla fine degli anni Quaranta, il «Cambridge Sentinel», in cui si encomia l'imprenditore americano per la sua decisione altruistica di raddoppiare gli stipendi a quegli operai che avrebbero coltivato "parsimonia, sobrietà e una vita salutare $»^{53}$. In questo modo egli poté così legittimare le proprie istanze razionaliste, ancorate su una divisione di genere garante dell'equilibrio produttivo e sociale.

\section{Perdita di stabilità: rifuggire dalla depravazione}

Come si è detto, la politica degli alti salari struttura una differenza di genere che si ripercuote all'interno della vita della classe operaia, razionalizzando così il lavoro produttivo. Ma ciò pone un problema notevole che possiamo sintetizzare attraverso le parole di Gramsci:

[...] l'alto salario è a due tagli: occorre che il lavoratore spenda «razionalmente» i quattrini più abbondanti, per mantenere, rinnovare e possibilmente per accrescere la sua efficienza muscolare-nervosa, non per distruggerla o intaccarla. Ed ecco la lotta contro l'alcool, l'agente più pericoloso di distruzione delle forze di lavoro, che diventa funzione di Stat $0^{54}$.

Quindi da una parte il lavoratore, ottenendo uno stipendio più elevato, ha la possibilità di spendere una parte del denaro in maniera diversa dalla semplice cura della famiglia. Ma dall'altra, la forte ripetitività del lavoro e l'alienazione di cui è vittima, spinge il lavoratore a spendere il proprio denaro in alcool, distruggendo così la stabilità ricercata da Ford.

L'attenzione posta da Ford e dalle sue ispezioni all'interno delle famiglie degli operai ${ }^{55} \mathrm{si}$ colloca in perfetta sintonia con il proibizionismo americano, dove si cerca di escludere dalla fabbrica e dall'intero sistema industriale i soggetti non adatti a quella civiltà delle buone maniere che l'industriale americano stava cercando di realizzare ${ }^{56}$. Pertanto, diventa rilevante tenere

\footnotetext{
${ }^{52}$ Ibidem, p. 414.

${ }^{53}$ «The Ford Altruism», in Cambridge Sentinel, XI, 28, 9 maggio 1914, p. 8.

${ }^{54}$ GRAMSCI, Antonio, op. cit., p. 2166.

${ }^{55}$ Ispezioni che continuarono ad essere fatte anche dopo la chiusura nei primi anni Venti del Sociological Department: FOOTE, Christopher L., WHATLEY, Warren C., WRIGHT, Gavin, «Arbitraging Discriminatory Labor Market: Black Workers at the Ford Motor Company, 1918-1947», in Journal of Labor Economics, 21, 3/2003, pp. 493-532, p. 504.

${ }^{56}$ Cfr. SETTIS, Bruno, op. cit., p. 78.
} 
conto di qualcosa a cui si è già accennato: all'interno delle fabbriche Ford si tenta di creare quello che Lewchuck definisce «club maschile», un fraternalismo operaio che rafforzi la mascolinità dei lavoratori, aiuti a sostenere il peso monotono della catena di produzione ${ }^{57}$, e che instilli nell'operaio l'idea che l'uomo debba anche badare alla propria famiglia, distogliendolo così dai vizi urbani. La donna viene esclusa da questo sistema connettivo fortemente virile tra gli operai e il datore di lavoro, diventando così elemento di antitesi rispetto alla mascolinità operaia.

A caratterizzare la mascolinità e la rispettabilità dell'operaio nella fabbrica Ford non era soltanto la sua capacità nel badare una famiglia sana ed equilibrata, ma anche sostenere i ritmi della linea di produzione, cioè diventare quell'«uomo nuovo» che per Gramsci non era altro che il «mass-produced man», ovvero "efficiente e intercambiabile» ${ }^{58}$. Chi non riusciva a tenere il passo, veniva declassato a ruoli «[adatti] alle donne», come il taglio dei nastri ${ }^{59}$. Si rende esplicito come il lavoro alla catena di produzione fosse il solo elemento caratterizzante per la mascolinità dell'operaio, mentre il resto delle mansioni in fabbrica venivano lasciati alle donne o agli afroamericani, ai quali venivano affidati i lavori più pesanti o umilianti ${ }^{60}$.

Ma l'alcool non è l'unico vizio di cui può cadere vittima l'operaio, giacché anche la «depravazione» sessuale rappresenta un altro forte elemento destabilizzante ${ }^{61}$, e la donna diventa così importante per la distruzione o la conservazione della moralità e della rispettabilità dell'operaio, ponendosi contemporaneamente come dispensatrice della stabilità della famiglia, così come possibile falla nel modello razionalizzato di Ford. Difatti, per quanto l'alcool costituisca un elemento fondamentale della lotta puritana del governo federale americano, in realtà la prostituzione ha costituito anch'essa un elemento di contrasto con le istanze morali della società statunitense.

La lotta contro la prostituzione fin dall'inizio si registra come uno sforzo legale e morale diretto verso la città, e ciò viene evidenziato dal medico Abraham Flexman che analizza i vizi sessuali come una vera e propria malattia urbana che cresce proporzionalmente all'estensione delle metropoli ${ }^{62}$. L'enorme flusso migratorio diretto verso l'America e proveniente dall'Occidente Europeo, aumentò ancora di più la convinzione presso l'opinione pubblica che la prostituzione stesse dilagando nelle città americane. Queste motivazioni giustificarono pertanto l'intervento delle autorità federali nel 1910, le quali cercarono definitivamente di criminalizzare la

\footnotetext{
${ }^{57}$ Cfr. LEWCHUK, Wayne A., op. cit., p. 847; diversamente la pensa Meyer, la quale sostiene che la ripetitività dell'attività lavorativa - che mette sotto sforzo le facoltà motorie e psicologiche dell'operaio - nei fatti abbia svirilizzato il lavoro: MEYER, Stephen, «Work, Play and Power, Masculine Culture on the Automotive Shop Floor, 1930-1960», in Men and Masculinities, 2/1999, pp. 115-134, pp. 120-121.

${ }^{58}$ CLARKE, Deborah, op. cit., p. 48.

${ }^{59}$ LEWCHUK, Wayne A., op. cit., p. 842.

${ }^{60}$ Cfr. SETTIS, Bruno, op. cit., pp. 82-83.

${ }^{61} \mathrm{Cfr}$. ibidem.

${ }^{62}$ Cfr. FELDMAN, Egnal, «Prostitution, the Alien Woman and the Progressive Imagination, 1910-1915», in American Quarterly, 19, 2/1967, pp. 192-206, p. 193.
} 
prostituzione $^{63}$, concentrando più che altro i propri sforzi verso la limitazione della tratta delle bianche. In realtà la questione della prostituzione rimase ambigua per molto tempo ancora, e molto spesso dall'opinione pubblica essa venne percepita come elemento "alieno" allo scenario puritano degli Stati Uniti, mentre il mercato di questi «atti immorali» coinvolse parecchie donne autoctone «vittime delle necessità economiche» ${ }^{64}$. In più non tutti erano d'accordo sulla completa criminalizzazione del fenomeno: per molto tempo l'ago della bilancia rimase proteso verso una silenziosa accettazione, giacché la prostituzione veniva sì rappresentata come un vizio animalesco e insalubre, ma «anche inevitabile» ${ }^{65}$.

Pertanto, quando non erano le stesse donne a rappresentare un enorme problema per la stabilità meccanizzata della struttura fordista, era la domesticity delle mogli a raffigurare il perfetto punto d'incontro tra il bisogno di stabilità del sistema e il controllo della moralità degli operai. In realtà quest'ultimo era un portato culturale che, come afferma Feldman, possiamo fare risalire ad una concezione in voga durante il periodo vittoriano, per cui è la donna la custode perfetta della «purezza sessuale», ponendosi così al di sopra delle «passioni della controparte maschile» ${ }^{66}$. In questo modo, come afferma Eileen Boris, «il genere e la sessualità non [furono] confinati all'interno delle mura domestiche», ma permearono all'interno della sfera delle rappresentazioni pubbliche ${ }^{67}$. Ma ciò non vuol dire che Ford sia stato un vero e proprio puritano; piuttosto egli rappresentava «quell'uomo che i Puritani delle origini temevano di più: l'apparente sostenitore della retta via che, privo di grazia, pecca contro la luce» ${ }^{68}$.

Primo tra tutti, Gramsci aveva compreso come le istanze puritane messe in atto da Henry Ford - così come da parte di molti industriali americani - non fossero sinceramente connesse ad un personale moralismo imperante. Esse non puntavano, infatti, a curarsi dell'«umanità» o della «spiritualità» dell'operaio, bensì erano tese a «conservare, fuori dal lavoro, un certo equilibrio psico-fisico che impedisca il collasso fisiologico del lavoratore, spremuto dal nuovo metodo di produzione $»^{69}$. Le donne garantiscono la stabilità di tale «equilibrio psico-fisico», rappresentate così come soggetti addetti alle cure domestiche e sessualmente passive di fronte alle pulsioni sessuali del marito. La sessualità coniugale della classe operaia diventa parte del processo di

\footnotetext{
${ }^{63}$ Prostituzione che però non aveva una definizione ben precisa, giacché non si rivolgeva soltanto al semplice atto sessuale a pagamento, ma ad "atti immorali" senza nessun'altra precisazione: DITMORE, Melissa [,] H., Prostitution and Sex Work, Santa Barbara, ABC-CLIO, 2011, p. 71.

${ }^{64}$ RIEGEL, Robert E., "Changing American Attitudes Toward Prostitution (1800-1920)», in Journal of the History of Ideas, 29, 3/1968, pp. 437-452, p. 450.

${ }^{65}$ SHUMSKY, Neil L., «Tacit Acceptance: Respectable Americans and Segregated Prostitution, 1870-1910», in Journal of Social History, 19, 4/1986, pp. 665-679, p. 665.

${ }^{66}$ FELDMAN, Egnal, op. cit., pp. 205-206.

${ }^{67}$ BORIS, Eileen, «The Gender of Labor History: The Difference It Makes», in Genesis, XV, 2/2016, pp. 147-166, p. 150. Qui - e nelle altre occorrenze che seguono - la traduzione è a cura dell'autore dell'articolo.

${ }^{68}$ BOSWORTH, David, «Idiot Savant: Henry Ford as Proto-Postmodern Man», in The Georgia Review, 54, 1/2000, pp. 11-39, p. 25.

${ }^{69}$ GRAMSCI, Antonio, op. cit., pp. 2165-2166.
} 
meccanizzazione del lavoratore, privata dei «colori "abbaglianti" dell'orpello romantico proprio del piccolo borghese e del bohémien sfaccendato» ${ }^{70}$. Pertanto, essa viene vista da Gramsci come una sessualità monogama e monotona costitutiva delle classi subalterne, la quale si oppone alla sfera sessuale delle classi elevate, compartecipe nella rappresentazione pubblica della sessualità dei lavoratori.

Come «il contadino che torna a casa la sera dopo una lunga giornata di fatica», l'operaio stremato dalla catena di produzione della Ford desidera la " "Venerem facile parabilemque" di Orazio», cioè passiva di fronte alle esigenze sessuali del marito, una donna che "non farà smancerie e non pretenderà la commedia della seduzione e dello stupro per essere posseduta» ${ }^{71}$. Ed ecco quindi che alla sfera privata e alla sfera pubblica si impongono due differenti sessualità: la stabilità sessuale della donna passiva all'interno delle mura domestiche contrapposta all'instabilità dei rapporti sessuali pubblici, ovvero da parte di donne che vendono il proprio corpo intaccando le istanze razionalizzatrici degli industriali. In questa sfera pubblica vi rientra anche la promiscuità sessuale delle classi elevate ma, diversamente dalla prostituzione ambiguamente combattuta dal sistema federale americano, quest'ultima non rappresenta un elemento pericoloso per la stabilità utopica voluta da Ford, giacché non interagisce direttamente con la classe degli operai vittima della regolamentazione sociale, familiare e lavorativa.

In realtà la pressione del lavoro meccanizzato, che per Taylor doveva rendere l'operaio un «gorilla ammaestrato» privo di capacità decisionali ${ }^{72}$, aveva privato i lavoratori non solo delle proprie energie, ma anche del proprio tempo, tanto che al ritorno a casa essi erano così esausti, sostenevano i detrattori di Ford, da non riuscire ad avere una vita sessuale attiva con le mogli. Inoltre l'industria, attraverso il Sociological Department, cerca di scoraggiare i divorzi, costringendo il marito a dover cedere gran parte dello stipendio alla moglie a separazione avvenuta $^{73}$. In questo modo si costruiva un modello familiare moralmente elevato per contrastare le distrazioni e i vizi della città, ma che contemporaneamente cercava di rendere fissi e stabili nel tempo i legami affettivi.

Tale struttura ci dà modo di poter meglio intendere che cosa sia per Gramsci la «quistione sessuale», e che cosa rappresenti in senso egemonico ${ }^{74}$ : una struttura che immagina se stessa come rigida, ma che impatta contro un sistema sociale che - come abbiamo visto - non sempre partecipa alla razionalizzazione delle vite degli operai, così come si confronta con una molteplicità umana che non sempre si lascia ridurre ad un unico modello. Essa quindi si palesa

\footnotetext{
${ }^{70}$ Ibidem, p. 2167.

${ }^{71}$ Ibidem.

${ }^{72}$ GRAMSCI, Antonio, op. cit., p. 2165.

${ }^{73}$ Cfr. LEWCHUK, Wayne A., op. cit., p. 844.

${ }^{74}$ Cfr. SLAUGHTER, Jane, "Gramsci's place in women's history», in Journal of Modern Italian Studies, 16, 2/2011, pp. 256-272, p. 263.
} 
come una superstruttura non rigida e non completamente efficiente nel plasmare $\mathrm{i}$ comportamenti degli individui chiamati a partecipare alle istanze produttrici della linea fordista. La pressione della pianificazione fordista tende a limitare la libertà decisionale degli operai, ponendola all'interno di una lotta manichea tra stabilità e instabilità del sistema.

Ma è lo stesso sistema che produce da sé quella instabilità che distrugge la propria impalcatura: il mercato automobilistico inizia a riferirsi anche alla controparte femminile nell'acquisto di automobili - che in pochi anni diverranno alla portata anche delle famiglie operaie $^{75}$. Come si vedrà nel paragrafo seguente, per quanto tale mercato cerchi di ricondurre le acquirenti ad un modello standardizzato di femminilità, legata necessariamente alla famiglia in quanto moglie ma soprattutto in quanto madre, in realtà sono proprio le macchine a dare una libertà parziale e un senso di autonomia alle housewifes. Le automobili, in ultima istanza, sono capaci di modificare parzialmente la "quistione sessuale», e se ne renderanno protagoniste proprio quelle donne rese fino ad allora passive di fronte alle pulsioni dell'«uomo nuovo».

\section{Il dilemma della libertà lockiana}

Ampliare il mercato automobilistico poneva le sue problematiche. Come evidenziato da Virgina Scharff, molti furono i critici che sottolineavano il pericolo di destinare delle automobili alle donne, poiché difficilmente controllabili dal punto di vista sociale, spaziale, sessuale ${ }^{76}$. Nella società americana la libertà dell'individuo è sempre stata rappresentata attraverso il movimento fisico in paesaggi sterminati, ovvero il mito - romantico e contemporaneamente morale - della frontiera successivamente messo in scena dal cinema hollywoodiano nei film western, ad esemplificazione di quello stato di natura lockiano parte della tradizione liberale statunitense ${ }^{77}$. Fin dall'inizio il settore pubblicitario ha cercato di utilizzare tale metafora come elemento cardine del movimento libero nello spazio e nel tempo, in uno scenario sempre più urbanizzato e capace di dare vita consumisticamente al sogno americano ${ }^{78}$.

Sebbene all'inizio tale mercato si riferisse a dei consumatori di sesso maschile, con il tempo si iniziò a pensare di dover produrre macchine pensate per le donne, dando vita ad una

\footnotetext{
${ }^{75}$ Tendenzialmente, dopo l'applicazione degli alti salari e i prestiti a basso interesse da parte di Ford verso i propri dipendenti, l'acquisto di una macchina diventa accessibile anche agli operai, specialmente dopo il 1916, quando il costo di una automobile Model T scende fino a $\$ 320$. Si veda in proposito DONKIN, Richard, The History of Work, New York, Palgrave Macmillan, 2010, pp. 146, 150.

${ }^{76}$ Cfr. SCHARFF, Virginia, Taking the Wheel: Women and the Coming of the Motor Age, New York, Free Press, 1991, passim.

${ }^{77}$ Cfr. GUIDIS, Catherine, op. cit., p. 64; e MEXAL, Stephen J., Two Ways to Yuma. Locke, liberalism, and western Masculinity in 3:10 to Yuma, in McMAHON, Jennifer, CSAKI, Steve B. (eds.), Philosophy and the western, New York, The University Press of Kentucky, 2010, pp. 69-87, p. 75.

${ }^{78}$ Cfr. GUIDIS, Catherine, op. cit., p. 64.
} 
pubblicizzazione che le legava a determinati ruoli di genere, che facevano riferimento ad una cultura patriarcale radicata all'interno dell'intera società statunitense. In realtà parte del mercato automobilistico, contrariamente alle industrie Ford, cercò di vendere alle donne non delle macchine con la stessa struttura meccanica delle automobili destinate ad un pubblico di soli uomini, ma limitanti dal punto di vista della mobilità. In questo senso alcune industrie iniziarono a immettere nel mercato le prime automobili elettriche, specifiche per un pubblico femminile, difficili da ricaricare e con una autonomia bassissima, tanto da dare alle donne a cui erano destinati tali veicoli solo l'illusione di essere libere nei propri spostamenti ${ }^{79}$. La Baker Electrics nel 1916 pubblicizzò la propria automobile elettrica all'interno della rivista «Life», mediante una locandina semplice nella propria rappresentazione di genere: il titolo dell'immagine, «Pleasure», sottolineava il fatto che alle donne non interessasse il "potere» o l'autonomia quanto il piacere ${ }^{80}$. Venne rievocata una immagine edenica che mette al centro della scena una donna con alle spalle l'automobile elettrica, circondata da fiori e farfalle, ma soprattutto da due bambine ${ }^{81}$. Qui emerge un elemento comune alle varie rappresentazioni pubblicitarie prese in esame: nella maggior parte dei casi la donna viene rappresentata nel ruolo di madre, quindi responsabile del proprio lavoro riproduttivo. L'armonia agreste della locandina rimane tale solo se la donna mantiene il ruolo di genitrice, rappresentandola così fuori dall'auto, seduta sul prato e a godere visivamente dei fiori ed escludendo la propria mobilità a vantaggio del proprio ruolo materno.

Da questo punto di vista la Ford opera in maniera diversa per quanto riguarda la vendita delle automobili: Henry Ford ha in mente di vendere quella che lui stesso definisce una "macchina universale", cioè facile da guidare anche per le donne ${ }^{82}$. Come si può vedere da uno dei pamphlet pubblicitari dell'industria del 1912, intitolato The Woman and the Ford si rende esplicito come la macchina Modello $\mathrm{T}$ sia così facile da guidare da essere fruibile anche dalle donne ${ }^{83}$. Analizzando il pamphlet, ci si rende conto di come si tenti di rafforzare l'identità domestica della donna. Anzi, l'acquisto della macchina combatte la monotonia di quelle donne che «hanno detto "vorrei essere un uomo"», mantenendo così intatta la loro identità di genere ${ }^{84}$.

Anche qui si fa riferimento ad un uso della macchina relativo al godimento della vita all'aperto, ma c'è qualcosa di più: sebbene possa sembrare un modo per rendere la donna autonoma, in realtà Ford «proponeva la macchina come una cura alla noia e alla insoddisfazione, non come fonte di potere ${ }^{85}$, dando loro uno sfogo temporaneo alle frustrazioni del proprio vincolo sociale e familiare ma, come si vede in altri pamphlet coevi, rafforzandone contemporaneamente il ruolo di

\footnotetext{
${ }^{79}$ Cfr. CLARKE, Deborah, op. cit., p. 19.

${ }^{80} \mathrm{Cfr}$. ibidem.

${ }^{81}$ Cfr. ibidem, fig.1, p. 20.

${ }^{82}$ Cfr. ibidem, p. 23.

${ }^{83}$ The woman and the Ford, Detroit, s.e., 1912, p. 7.

${ }^{84}$ Ibidem, p. 11.

${ }^{85}$ CLARKE, Deborah, op. cit., p. 16.
} 
madre e moglie ${ }^{86}$. Anche se il Modello $\mathrm{T}$ si proponeva come «una vera e propria arma per cambiare l'ordine [sociale] $»^{87}$, in realtà si rendeva intatta l'identità di genere nelle proprie rappresentazioni di marketing. Sebbene siano state pubblicizzate talune locandine rappresentanti delle donne nel ruolo di lavoratrici ${ }^{88}$, ancora quindici anni dopo, la Ford insistette sulla rappresentazione delle automobiliste in funzione di madre. Come si può vedere dalla locandina pubblicitaria dell'industria del 1925, che invoglia ad acquistare l'automobile grazie ai «piccoli pagamenti» mensili, ci si ritrova nuovamente di fronte ad una donna nel ruolo di madre, con alle spalle la Modello T, e con due bambini al suo seguito ${ }^{89}$; in testa al manifesto troviamo lo slogan «Mother need a Ford, Buy It Now». È quindi chiaro che l'autonomia immaginata da Ford per le acquirenti è molto limitata e si riferisce a quell'equilibrio sociale che l'industriale stava cercando di creare per rendere produttiva l'intera struttura economica della fabbrica.

Pertanto, il mercato automobilistico simbolicamente offriva alla donna la possibilità di essere autonoma, ma non concretamente. Dal punto di vista culturale la società americana iniziò comunque a porsi problematicamente nei confronti delle donne in automobile. Se da una parte sottolineava l'incapacità del "gentil sesso" nella guida - come implicitamente affermato dalla Ford e dai suoi pamphlet - dall'altra si iniziò a discutere sulla loro sessualità ${ }^{90}$. Perché se l'intento delle industrie automobilistiche, specialmente della Ford, era quello di ampliare alla sfera femminile il mercato delle automobili, continuando a limitare le donne all'interno dei loro ruoli di genere stabilitisi socialmente, quello che non potevano sapere è che in realtà diedero loro un mezzo che almeno in potenza - si dimostrava capace di affrancarle dai limiti a loro culturalmente imposti. Perché l'automobile non significava soltanto potenza tecnologica, ma «autonomia individuale» oltre che «velocità, tempo e direzione»" ${ }^{91}$.

Insieme alla vendita delle automobili, si dà quindi modo alle donne di esplorare la propria sessualità in maniera diversa da come prescritto dalla stabilità fordista: come registra uno studio di due sociologi della fine degli anni Venti in una città dell'Indiana, Middletown, le macchine iniziarono ad essere uno dei luoghi preposti per alcune delle esperienze sessuali della vita delle ragazze, diventando per alcuni nemiche «della casa e della società»" ${ }^{92}$ Sebbene alcune voci affermassero che Ford, mosso dal suo spirito conservatore, stesse cercando di creare dei sedili posteriori adatti ad evitare questi atti indecenti, la realtà dei fatti è che quella che era stata data

\footnotetext{
${ }^{86}$ Cfr. ibidem, p. 22.

${ }^{87}$ The woman and the Ford, cit., p. 3.

${ }^{88}$ Cfr. CLARKE, Deborah, op. cit., p. 24.

${ }_{89}$ Mother Needs a Ford, s.l., s.e., 1925, URL: < https://www.thehenryford.org/collections-andresearch/digital-collections/artifact/340304 > [consultato il 26 giugno 2017].

${ }^{90} \mathrm{Cfr}$. BERGER, Michael L., The Automobile in American History and Culture: A Reference Guide, Westport-London, Greenwood Press, 2001, p. 151.

${ }^{91}$ CLARKE, Deborah, op. cit., p. 12.

${ }^{92}$ LYND, Robert S., LYND, Helen M., Middletown. A Study in Modern American Culture, New York, Harcourt Brace, 1929, p. 258.
} 
come una libertà in potenza, diventò libertà in atto ${ }^{93}$. Nacque anche un genere letterario scritto da donne e che iniziò a mettere in luce la loro relazione con le automobili, ma non tanto dal punto di vista sessuale quanto da quello relativo all'avventura e alla libertà di movimento delle automobiliste ${ }^{94}$.

Ed ecco che una struttura come quella voluta da Ford, pensata per essere, con le parole di Nancy Fraser, totalizzante ${ }^{95}$, iniziò a generare da sé stessa quell'elemento destabilizzante capace di distruggere la sua perfezione utopica, lasciando alla società americana il compito di limitare le istanze morali delle classi operaie e, soprattutto, delle donne.

\section{Conclusioni}

Come aveva capito Gramsci, l'americanismo di cui era permeato il fordismo poteva rivendicare quella «formuletta» di Giovanni Gentile che poneva una «filosofia che non si enunzia in formule ma si afferma nell'azione», perché non modificava soltanto l'uomo ma anche la «realtà esterna», la «cultura $»^{96}$. In un certo senso, quindi, il fordismo aveva cercato di strutturare la relazione con la classe operaia a partire dalla realtà culturale americana, modificandola a proprio vantaggio, come nel caso della lotta contro gli alcolici in epoca proibizionista. Ma il fordismo impattò violentemente contro una crisi economica che fece crollare la politica degli alti salari ${ }^{97}$ - colonna portante del disciplinamento della classe degli operai - e soprattutto si scontrò contro una realtà sociale in continuo movimento e mai uguale a se stessa. Dalla creazione del Modello T (1909) alla crisi economica del triennio 1919-21, l'industria automobilistica visse varie cesure, così come la società americana: dallo scoppio della Prima guerra mondiale e l'entrata degli Stati Uniti nel conflitto, con la conseguente decisione dell'industriale Henry Ford di accettare le commesse militari $^{98}$, alla decisione dell'azienda di chiudere il Sociological Deparment ${ }^{99}$.

In questo senso le operaie e il loro rapporto con l'industria non cambia sempre grazie alle istanze della razionalizzazione fordista, ma anche in base al mutamento storico della società americana. Le rappresentazioni delle donne generate dall'industria Ford non sono che una delle tante sfaccettature di una società americana che si evolve, rimanendo per molto tempo patriarcale e fortemente maschilista. La mancata analisi di genere da parte di molti studiosi del sistema fordista è stata probabilmente generata dall'incapacità di pensare a tale sistema come

\footnotetext{
${ }^{93}$ Cfr. CLARKE, Deborah, op. cit., p. 28.

${ }^{94}$ Cfr. ibidem, p. 26.

${ }^{95}$ Cfr. FRASER, Nancy, op. cit., p. 163.

${ }^{96}$ GRAMSCI, Antonio, op. cit., p. 2152.

${ }^{97}$ Cfr. SETTIS, Bruno, op. cit., p. 301.

${ }^{98} \mathrm{Cfr}$. ibidem.

${ }^{99}$ FOOTE, Christopher L., WHATLEY, Warren C., WRIGHT, Gavin, op. cit., p. 500.
} 
parte integrante di un vero e proprio discorso sulle donne, poiché visto e pensato come esclusivamente maschile.

La rilettura del fordismo è avvenuta - come si è già detto - solo molto dopo, quanto ci si è interrogati sul postfordismo e i rapporti di genere, snaturandone anacronisticamente alcuni degli elementi più importanti. A 80 anni dalla morte di Gramsci, si è qui voluto tentare di rendere evidente come egli abbia delineato nei suoi Quaderni una metodologia analitica ancora valida per potere studiare il passato, capace di osservare ed esaminare le strutture sociali/culturali/politiche dalle proprie rappresentazioni interne. In questo senso, l'analisi di genere che qui si è proposta non sarebbe stata possibile senza un approccio gramsciano al concetto di fordismo. 


\section{L'AUTORE}

Bruno Walter Renato TOSCANO è attualmente studente del corso di Laurea Magistrale in Scienze Storiche presso l'Università di Bologna, indirizzo storia contemporanea. Ha conseguito la laurea triennale con il massimo dei voti in Studi Filosofici e Storici (L-5) presso l'Università degli Studi di Palermo, discutendo una tesi in storia contemporanea dal titolo George L. Mosse: la nuova politica per l'uomo nuovo, relatore Salvatore Lupo.

URL: < http://www.studistorici.com/progett/autori/\#Toscano > 